\title{
Women in the field of oral and maxillofacial surgery- a difficult road
}

\section{Editorial}

Woman in society is constantly between law and tradition and their influence on her fate. Sometimes law may guarantee equal rights for men and women but tradition could be so strong that weakens the impact of a reasonable law. In 1979, I participated in the entrance exams, similar to my male classmates, and entered dental school at Tehran University with a high rank. That time it was a dominant opinion that dentistry is very difficult for women and if a woman is entering the field, she must work with a male colleague. But in college I observed that although number of women is less than men, but sometimes they were better than men in dental procedures. In fact, it was the person's potential and individual effort that was resulting in superiority in the field. Nearly 40 years has passed since then. State and gender composition of the dental faculty in our country has totally changed and in specialized and general dentistry, many female dentists play proper and worthy role and society's attitude to the role of women in this area is changing. In quantitative terms, while the number of male dentists is more than the number of women, the proportion of women has quickly changed and now in some colleges, the number of female students is more than male students. But in oral and maxillofacial surgery, the situation is different. The percentage of entered women in this field is still very low and people face female maxillofacial surgeons with disbelief. Articles in international journals about the role of women in oral and maxillofacial surgery show the degree of importance of this issue for the authors of the articles that are from professional people in oral and maxillofacial surgery. In our country there aren't legal barriers for women entering to the field of oral and maxillofacial surgery and there is equal probability for entering and working in this field for both genders. However, previously, very low number of women was entering the field. In 2013, the number of female oral and maxillofacial surgeons with respect to male surgeons in Iran was 5\%. On one hand, this is due to the difficulty of the major itself, and on the other hand, it is because of the implicit assumption of special responsibility for family (like motherhood and major and minor home affairs) on behalf of the community for women. In these conditions, to compensate for this heavy responsibility, it is necessary to allocate a certain percentage from entrance to surgery field in all medical majors to women. In addition to family responsibilities and raising children, psychological pressures and non-professional nature is also coming from some people in education and work due to their cultural and social problems, which has a negative role on women's social development, especially in the sensitive responsibilities. My personal experience as a graduate from another country is remarkable, where after returning to my country, I inevitably had to spend a course called "Supplemental Course in Oral and Maxillofacial Surgery" at Tehran University (2004-2007).

For me, none of student rights specified by law and educational programs helped and I was deprived of many clinical routine training facilities by department head. In compliance with the discriminative policy of the department authorities, almost all residents, which all were males, ran this policy. At the same time, another man colleague
Volume 6 Issue 4 - 2017

Mirmohammadi mitra
Oral \& Maxillofacial Surgeon, Iran

Correspondence: Mirmohammadi Mitra, Oral \& Maxillofacial Surgeon,Iran, Email mitra_mirmohammadi@yahoo.com

Received: February 17, 2017| Published: February 21, 2017

who came from another country, spent this period without any problems. What allows the pressure and discrimination against women in educational centers is the lack of deterrent laws to defend women in such circumstances. I finished my course by relying on the law, but unfortunately, these individuals were not punished for their illegal activities against me and for wasting my limited academic time which should've been spent on academic work and clinical improvement. In the field of oral and maxillofacial surgery, in addition to the treating aspect, in order to achieve excellent results, apart from having scientific support, the surgeon needs to have talent, and qualitative and quantitative growth of women makes it essential for promoting oral and maxillofacial surgery. Each elimination method for keeping them from the field is not only oppression to female surgeons but also is oppression to oral and maxillofacial surgery. Currently we have about 400 maxillofacial surgeons in Iran. So far, except me, who was a board member of civil society (scientific association of Oral and Maxillofacial Surgeons in Iran) in prior period, none of the female maxillofacial surgeons were in the leadership of this association. Despite the fact that almost all graduated women which work in academic departments are very good professors, none of them has a key management position, or if they have, from official's kindness, these positions have been assigned for them only temporarily. Although I suffered very much in graduate school as a woman, but these pressures didn't hinder my scientific and social activities. Just I should overcome lots of barriers. I have published a magazine in the field of oral and maxillofacial surgery for 4 years. This is the only monthly scientific magazine in the field of oral and maxillofacial surgery in Iran and shows that superiority is not defined by gender. The merit is determined by person's work and activity. However, as I mentioned, acts of harassment and domination by men, could marginalize women and hinder their talent. Despite this fact that in our country, many male colleagues assert that they believe in human rights standards and have respect for women rights, but sometimes in some situations, some people abuse the society customs against women and can create an environment that would not leave any room for other opinion. I think these reasons are sufficient for women to give up the professional and social activities and work in the far corner away from the harm of discrimination and threats to their jobs. I think that for achieving fair conditions, the laws for recognizing the legal 
rights of women are not sufficient; changing the culture and attitudes for law enforcement and equal rights of human observance is also necessary. Achieving this requires many civil efforts.

\section{Funding}

None.

\section{Acknowledgments}

None.

\section{Conflicts of interest}

The authors declare that there is no conflict of interest. 